\title{
Structural model for the first wall W-based material in ITER project
}

\author{
Dehua Xu • Xinkui He - Shuiquan Deng • \\ Yong Zhao
}

Received: 2 December 2013/Revised: 23 April 2014/Accepted: 26 April 2014/Published online: 23 May 2014

(C) The Author(s) 2014. This article is published with open access at Springerlink.com

\begin{abstract}
The preparation, characterization, and test of the first wall materials designed to be used in the fusion reactor have remained challenging problems in the material science. This work uses the first-principles method as implemented in the CASTEP package to study the influences of the doped titanium carbide on the structural stability of the W-TiC material. The calculated total energy and enthalpy have been used as criteria to judge the structural models built with consideration of symmetry. Our simulation indicates that the doped $\mathrm{TiC}$ tends to form its own domain up to the investigated nano-scale, which implies a possible phase separation. This result reveals the intrinsic reason for the composite nature of the W-TiC material and provides an explanation for the experimentally observed phase separation at the nano-scale. Our approach also sheds a light on explaining the enhancing effects of doped components on the durability, reliability, corrosion resistance, etc., in many special steels.
\end{abstract}

Keywords Tungsten-based material · First wall material · First principles

D. Xu $\cdot$ X. He $\cdot$ S. Deng $(\bowtie) \cdot$ Y. Zhao

Key Laboratory of Magnetic Levitation Technologies and

Maglev Trains, Ministry of Education of China, and

Superconductivity and New Energy R\&D Center, Southwest

Jiaotong University, Chengdu 610064, China

e-mail: s.deng@swjtu.edu.cn

D. $\mathrm{Xu}$

School of Physical Science and Technology, Southwest Jiaotong

University, Chengdu 610064, China

\section{Introduction}

In facing the imminent depletion of fossil fuels and the mounting demand of energy resources, various new sustainable energy resources have been proposed. Among which, the fusion energy has been considered the only way to solve the constantly growing demands of energy and the increasingly stringent requirements of environment. The ITER (International Thermonuclear Experimental Reactor) project is one important step for humankind to harness the fusion energy [1]. The first wall material is crucially important in the plasma facing components, which work in an extreme environment exposing to high energy particles and heat flux from the plasma [2]. Roughly speaking, the first wall materials can be classified into low $\mathrm{Z}$ materials and high $\mathrm{Z}$ materials. Tungsten belongs to the high $\mathrm{Z}$ category, which has the advantage of high strength at high temperature, and good resistance against erosion and irradiation due to high energy particles such as neutrons, He, etc. However, pure tungsten has a low fracture toughness which can be improved by changing its chemical composition through alloying or sintering with low $\mathrm{Z}$ materials to form composite materials. In the last few years, many efforts have been made in studying the tungsten-based materials such as $\mathrm{W}-(0.3,0.9,1.7) \mathrm{TiC}(\mathrm{wt} \%), \mathrm{W}-(0.3$, $1.0,2.0) \mathrm{Y}$, and $\mathrm{W}-(0.3,1.0,2.0) \mathrm{Y}_{2} \mathrm{O}_{3}$, etc. [3, 4].

The W-TiC system was first discovered and systematically studied by the research group of Kurishita. They have not only studied the preparation conditions, in particular, the effects of $\mathrm{Ar}, \mathrm{N}_{2}, \mathrm{H}_{2}$, etc., atmosphere on the final mechanical properties [5-8], but also have shown that the addition of $\mathrm{TiC}$ can result in significant refinement of grains of the alloys, which can thus enhance the ductility and resistance to the neutron irradiation. In addition, the high temperature mechanic properties and the crack and 
fracture mechanical properties can also be improved. [913]. The possible reason as suggested by Kurishita et al. [14] is that the addition of $\mathrm{TiC}$ and the recrystallization of $\mathrm{W}-\mathrm{TiC}$ alloy result in much efficient packing of the grains and strengthening grain boundaries. The other research activities [15-18] have also confirmed that the final properties of this system depend heavily on the preparation routes and details, a phenomenon indicating the role of multi-scale microstructures. Although many experimental works have been done on this system, the microscopic mechanism which connects the multi-scale structures and the properties has not been established. So far as we know, there has been hitherto no theoretical work done on $\mathrm{W}-\mathrm{TiC}$ system. This work focuses on the inhomogeneity at the nano-scale and attempts to understand the experimental findings from the electronic structures.

\section{Computational details}

In this work, we have used the state-of-the-art quantum code CASTEP for all computations. In our calculations, the density functional theory (DFT) is approximated using local density approximation (LDA) and its generalized gradient corrections (GGA) as implemented in the PBE formulation [19]. The built-in ultra-soft pseudo-potentials have been used throughout this work to treat the core electrons, which leads to a relatively low energy cutoff of $310.0 \mathrm{eV}$ for plane wave expansions of wave functions, potentials, etc. A $4 \times 4 \times 8 \mathrm{k}$-mesh in the first Brillouin zone is used for all integrations in the momentum space. Throughout this work, the DFT total energy, enthalpy if external pressure is present, has been taken as the object function for geometry optimization with respect to each test structure model. The high-dimensional multivariable minimization problem is solved using the BroydenFletcher-Goldfarb-Shanno (BFGS) quasi-Newton scheme [20].

\section{Initial structure models}

The experimental researches on tungsten-based materials have studied a wide range of composition for $\mathrm{TiC}$ and other chemicals. In this work, we investigate a specific composition, $\mathrm{W}+\sim 3 \% \mathrm{TiC}$ in weight percentage, which corresponds to a mole composition of $\mathrm{W}_{7}(\mathrm{TiC})$. A supercell technique is used to study the possible microstructures at the nano-length scale. As shown in Fig. 1b, a basic supercell of $2 \times 2 \times 1$ with respect to that of the $\alpha-W$ (Fig. 1a) contains $8 \mathrm{~W}$ atoms.

According to the symmetry of the supercell as shown in Fig. 1b, we have built four different models as shown in
Fig. 2. In Fig. 2 (a) the Ti atom is put on the edge of the supercell, while the $\mathrm{C}$ atom is put inside the supercell, but at an asymmetric position to the two $\mathrm{Ti}$ atoms ( $\mathrm{Ti}-\mathrm{C}$ : $2.162 \AA, 2.390 \AA$ ); (b) Ti atom is put at the center position of the $\alpha-\mathrm{W}$ with $\mathrm{Ti}$ and $\mathrm{C}$ atoms (Ti-C: $2.162 \AA$ ) lying in the $(001)$ plane but not in the $(010)$ plane in contrast to that in model (c); (c) Both $\mathrm{Ti}$ and $\mathrm{C}$ atoms are put on the (001) surface of the supercell with Ti-C distance at $2.162 \AA$ and Ti, C atoms lying in (010) plane; (d) Same as for (c) except that the $\mathrm{C}$ atom lies equally distant (Ti-C: $2.162 \AA$ ) to the two $\mathrm{Ti}$ atoms.

\section{Results and discussion}

The optimization on model (d) results in a configuration with the minimum energy as compared to the other three models. The optimization starting from the other three models lead to higher energy and larger stresses. Considering the periodicity of the models, model (a) and (d) are actually very similar, while models (b) and (c) belong to one class. Our calculations indeed obtain almost the same geometrical details for models (b) and (c). In both cases, the calculated Ti-C distance is $1.974 \AA$, which is a little shorter than that, $2.162 \AA$, in the TiC structure. The next Ti-C distance in both models is $3.709 \AA$, which clearly shows that the $\mathrm{Ti}-\mathrm{C}$ units in both models remain isolated with each other. The optimized $\mathrm{W}-\mathrm{C}$ distance in (b) and (c) is $2.004 \AA$, shorter than that in the WC structure (2.197 $\AA$ ). The separation of TiC units with each other may cost more energy for the $\mathrm{W}$ host structure to accommodate such units. Our calculations indicate that models (b) and (c) are both energetically unfavorable by an energy of about $0.23 \mathrm{eV}$ higher than that of model (d). As we mentioned above, models (a) and (d) are structurally very similar. They differ with each other only in the locations of the $\mathrm{C}$ atoms. In model (d) the $\mathrm{Ti}-\mathrm{C}$ units lie in the $(010)$ plane while those in model (a) cross the (010) plane. In these two structures, the $\mathrm{TiC}$ forms zigzag chains which run along the $\mathrm{c}$ direction. This character is similar to that observed in the crystal of TiC. For model (a), the geometric optimization shifts the two Ti atoms away from the (010) W plane, while the original longer Ti-C distance, $2.392 \AA$, in the zigzag chain becomes $1.912 \AA$, with the other $\mathrm{Ti}-\mathrm{C}$ distance almost unchanged. For model (d), the basic geometrical character remains unchanged except that the Ti-C distance $(2.221 \AA$ ) becomes longer than that in the initial model. The optimized W-C distance in model (d) is $1.997 \AA$ which is shorter than the normal $\mathrm{W}-\mathrm{C}$ bond length. This fact indicates a stronger $\mathrm{W}-\mathrm{C}$ bonding, which thus weakens the $\mathrm{Ti}-\mathrm{C}$ bonding and results in a longer $\mathrm{Ti}-$ C distance (2.221 $\AA$ ). The energy differences between the models in each category are negligibly small, for example, 


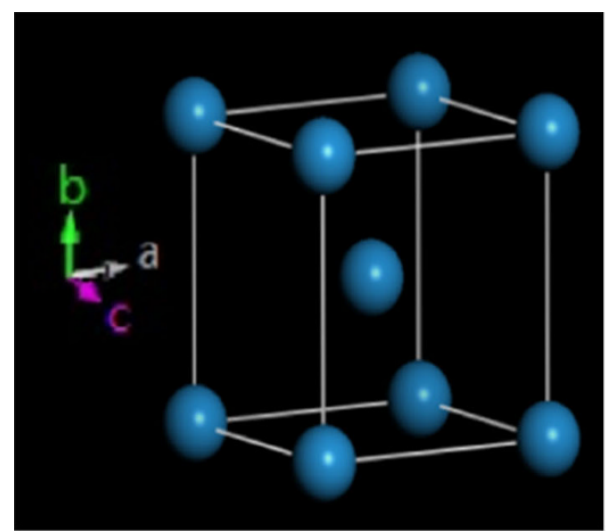

(a)

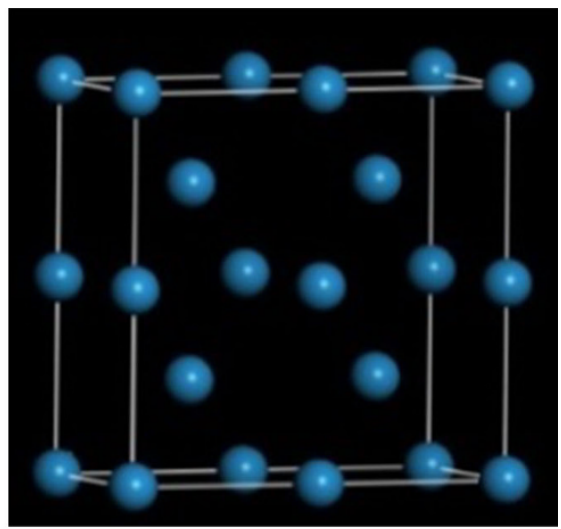

(b)

Fig. 1 a The unit cell of $\alpha-W$; $\mathbf{b}$ the $2 \times 2 \times 1$ supercell of $\alpha-W$, the coordinate system is for both figures

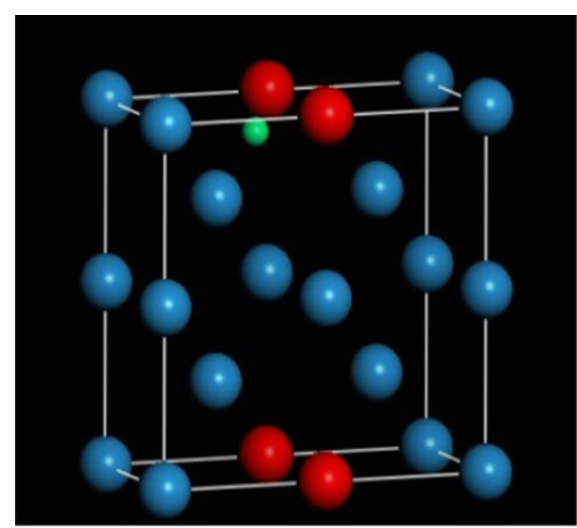

(a)

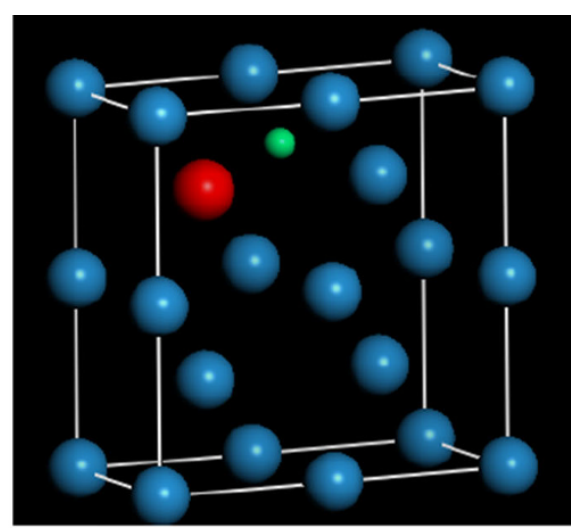

(b)

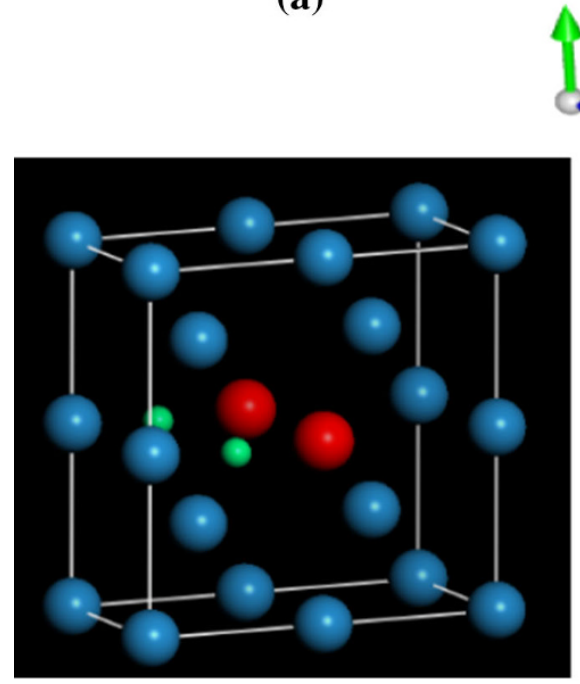

(c) b

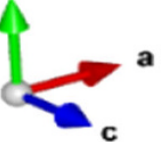

a

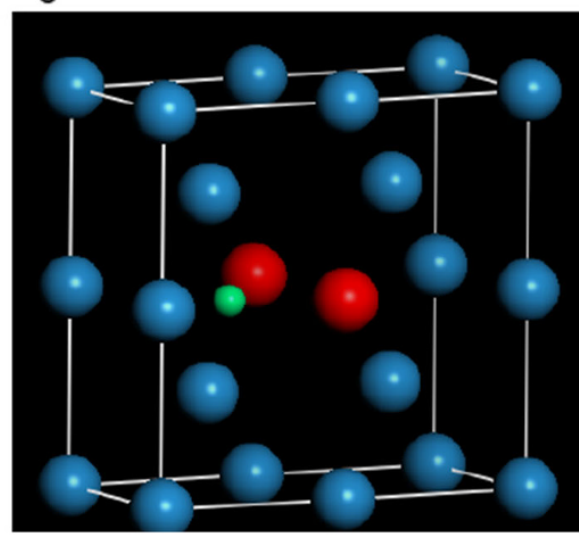

(d)

Fig. 2 In a-d, the blue balls represent W, red balls represent Ti, and green balls represent $\mathrm{C}$. The crystallographic coordinate system shown in the center is for all models from a to $\mathbf{d}$

the energy difference between (b) and (c) is only $0.0418 \mathrm{eV}$, which is at the order of phonon energy. In all the cases, the $\mathrm{Ti}-\mathrm{C}$ bonding is weakened due to the formation of $\mathrm{W}-\mathrm{C}$ bonding, which indicates that the $\mathrm{W}-$ TiC composite materials are not pure physical mixing, chemical interactions occur in all the cases. Nevertheless, 
this $\mathrm{W}-\mathrm{C}$ bonding is not large enough to compensate the energy lost due to the weakening of Ti-C bonding. As we have described above that the configurations (a, d) which keep the character of $\mathrm{TiC}$ structure have indeed energy advantage. This energy gain favors the phase separation at least at the unit-cell length that we investigated. We thus have given a theoretical explanation to the observed experimental fact. [10, 21].

Recently, we have used more accurate VASP code [22, 23 ] to calculate the above models as well as much larger models. When the chosen models are large enough to be comparable with the experimental $\mathrm{W}-\mathrm{TiC}$ ratio, the $\mathrm{Ti}-\mathrm{C}$ distance converges to a value of $\sim 2.16 \AA$. This is consistent with the result obtained by CASTEP code. The full analyses on results of VASP calculations are undergoing and have not been finished.

It has already been well known that some tiny amounts of elements such as C, Mn, Si, P, S, Cr, Mo, V, etc., play essential roles in enhancing the hardness, durability, fracture toughness, and good thermal performance of some special steels under extreme weather conditions [24]. A typical example is the widely used rail-track steel which contains $\sim 0.4 \%-0.8 \% \mathrm{C}, \sim 0.65 \%-1.7 \% \mathrm{Mn}, \leq 0.6 \%$ $\mathrm{Si}$, and other trace elements depending on the type of the steels. However, as in the $\mathrm{W}-\mathrm{TiC}$ case, the microscopic mechanisms which depend on the multi-scale structures of the interested composite materials remain unsolved. The recent experimental and theoretical studies [25-27] have shown the toughness of the problem. Our first-principles approach as used in this work provides a tool to attack the problem at the atomic and subatomic length scales. Because of its universality, we do expect its important applications in studying complex extreme fusion materials as well as the railway track materials.

\section{Conclusion}

Through using the first-principle method as implemented in the CASTEP code, we have optimized four models which simulate the relative configurations of $\mathrm{TiC}$ in the $\mathrm{W}$ lattice. By using the total energy and enthalpy as the standard, we have analyzed the obtained results. We conclude that although there exists $\mathrm{W}-\mathrm{C}$ interactions which weaken the Ti-C bonding, the Ti-C units tend to stay together to keep the structural character in the $\mathrm{TiC}$ structure. This provides an explanation for the experimental phenomenon of phase separation between $\mathrm{W}$ and $\mathrm{TiC}$ at nano-scale. However, we must point out that our conclusion is based on the relative smaller models which limit the choice of the supercell. Besides, our models involve only the chain configuration and the two atom Ti-C unit, therefore, it is worth further studies on more and larger realistic models to test the reliability of the conclusions that we drawn here.

Acknowledgments This work is finantially supported by the Science Foundation for International Cooperation of Sichuan Province (2014HH0016), the Fundamental Research Funds for the Central Universities (SWJTU2014: A0920502051113-10000), National Magnetic Confinement Fusion Science Program (2011GB112001).

Open Access This article is distributed under the terms of the Creative Commons Attribution License which permits any use, distribution, and reproduction in any medium, provided the original author(s) and the source are credited.

\section{References}

1. Barabash V (2007) Materials challenges for ITER-current status and future activities. J Nucl Mater 367:21-32

2. Federici G, Wuerz H, Janeschitz G et al (2002) Erosion of plasma-facing components in ITER. Fusion Eng Des 61:81-94

3. Baluc N, Schäublin R (2011) From materials development to their test in IFMIF: an overview. Nucl Fusion 51(11):113-116

4. Rieth M, Dudarev SL (2013) Recent progress in research on tungsten materials for nuclear fusion applications in Europe. J Nucl Mater 432(1-3):482-500

5. Ishijima Y, Kurishita H, Yubuta K et al (2004) Current status of ductile tungsten alloy development by mechanical alloying. J Nucl Mater 329-333, Part A(0):775-779

6. Kajioka M, Sakamoto T, Nakai K et al (2011) Effects of plastic working and MA atmosphere on microstructures of recrystallized W-1.1\% TiC. J Nucl Mater 417(1-3):512-515

7. Kawai M, Kurishita H, Kokawa $H$ et al (2012) Development of advanced materials for spallation neutron sources and radiation damage simulation based on multi-scale models. J Nucl Mater 431(1-3): 16-25

8. Matsuo S, Kurishita H, Arakawa H et al (2008) Deformability enhancement in ultra-fine grained, Ar-contained $\mathrm{W}$ compacts by TiC additions up to 1.1\%. Mater Sci Eng A 492(1-2):475-480

9. Kitsunai Y, Kurishita H, Kayano H et al (1999) Microstructure and impact properties of ultra-fine grained tungsten alloys dispersed with TiC. J Nucl Mater 271-272:423-428

10. Kurishita H, Amano Y, Kobayashi S et al (2007) Development of ultra-fine grained $\mathrm{W}-\mathrm{TiC}$ and their mechanical properties for fusion applications. J Nucl Mater 367-370, Part B(0):1453-1457

11. Kurishita H, Kobayashi S, Nakai K et al (2008) Development of ultra-fine grained $\mathrm{W}-(0.25-0.8) \mathrm{wt} \% \mathrm{TiC}$ and its superior resistance to neutron and $3 \mathrm{MeV} \mathrm{He-ion} \mathrm{irradiations.} \mathrm{J} \mathrm{Nucl} \mathrm{Mater}$ 377(1):34-40

12. Kurishita H, Matsuo S, Arakawa $H$ et al (2008) Superplastic deformation in $\mathrm{W}-0.5 \mathrm{wt} . \%$ TiC with approximately $0.1 \mu \mathrm{m}$ grain size. Mater Sci Eng A 477(1-2):162-167

13. Kurishita H, Matsuo S, Arakawa $\mathrm{H}$ et al (2009) High temperature tensile properties and their application to toughness enhancement in ultra-fine grained $\mathrm{W}-(0-1.5) \mathrm{wt} \%$ TiC. J Nucl Mater 386-388:579-582

14. Kurishita H, Matsuo S, Arakawa H et al (2010) Development of re-crystallized $\mathrm{W}-1.1 \% \mathrm{TiC}$ with enhanced room-temperature ductility and radiation performance. $\mathrm{J}$ Nucl Mater 398(1-3):87-92

15. Genç A, Coşkun S, veçoğlu ML (2010) Microstructural characterizations of $\mathrm{Ni}$ activated sintered $\mathrm{W}-2 \mathrm{wt} \% \mathrm{TiC}$ composites 
produced via mechanical alloying. J Alloy Compd 497(1-2):80-89

16. Genç A, Coşkun S (2010) M.L. veçoğlu. Decarburization of TiC in Ni activated sintered W-xTiC $(\mathrm{x}=0,5,10,15 \mathrm{wt} \%)$ composites and the effects of heat treatment on the microstructural and physical properties. Int $\mathrm{J}$ Refract Metal Hard Mater 28(3):451-458

17. Matsuo S, Kurishita H, Arakawa $H$ et al (2008) Deformability enhancement in ultra-fine grained, Ar-contained W compacts by TiC additions up to $1.1 \%$. Mater Sci Eng A 492(1-2):475-480

18. Chen Y, Wu YC, Yu FW et al (2008) Microstructure and mechanical properties of tungsten composites co-strengthened by dispersed $\mathrm{TiC}$ and $\mathrm{La}_{2} \mathrm{O}_{3}$ particles. Int $\mathbf{J}$ Refract Metal Hard Mater 26(6):525-529

19. Perdew JP, Burke K, Ernzerhof M (1996) Generalized gradient approximation made simple. Phys Rev Lett 77(3):865-868

20. Shanno DF (1978) Conjugate gradient methods with inexact searches. Math Oper Res 3(2):244-256
21. Veleva L (2011) Contribution to the production and characterization of $\mathrm{W}-\mathrm{Y}, \mathrm{W}-\mathrm{Y}_{2} \mathrm{O}_{3}$ and $\mathrm{W}-\mathrm{TiC}$ materials for fusion reactors. [D] Lausanne Ecole Polytechnique Federale de Lausanne

22. Kresse G, Joubert D (1999) From ultrasoft pseudopotentials to the projector augmented-wave method. Phys Rev B 59:1758

23. Kresse G (2007) Vienna ab-initio simulation package (VASP), http://cms.mpi.univie.ac.at/vasp/vasp/vasp.html

24. ArcelorMittal Products Information (2013) http://www.arcelormi 'ttal.com/rails specialsections/en/

25. Missori S, Sili A (2000) Structural characterization of C-Mn steel laser beam welded joints with powder filler metal. Weld Res Suppl 79(11):317-323

26. Song R, Ponge D, Rabbe D (2005) Influence of Mn content on the microstructure and mechanical properties of ultrafine grained $\mathrm{C}-$ Mn Steels. ISIJ Int 45(11):1721-1726

27. Eden HC, Garnham JE, Davis CL (2007) Influential microstructural changes on rolling contact fatigue crack initiation in pearlitic rail steels. Mater Sci Technol 21(6):623-629 